\title{
Impulsive Control of the Rotor-Stator Rub Based on Phase Characteristic
}

\author{
Jieqiong Xu, ${ }^{1}$ Qunhong Li, ${ }^{1}$ and Shimin Wang ${ }^{2}$ \\ ${ }^{1}$ College of Mathematics and Information Science, Guangxi University, Nanning 530004, China \\ ${ }^{2}$ The Research Lab of Dynamics and Control, Beijing University of Aeronautics and Astronautics, Beijing 100091, China
}

Correspondence should be addressed to Jieqiong Xu; clh4@163.com

Received 1 December 2013; Accepted 17 February 2014; Published 25 March 2014

Academic Editor: Muhammad Usman

Copyright (c) 2014 Jieqiong Xu et al. This is an open access article distributed under the Creative Commons Attribution License, which permits unrestricted use, distribution, and reproduction in any medium, provided the original work is properly cited.

An impulsive control method is proposed to eliminate the rotor-stator rubbing based on the phase characteristic. The relation between the vibration energy and the phase difference suggests the starting point for controlling the rotor-stator rubbing by implementing impulse. When the contact between the rotor and the stator occurs, the impulse is implemented in $x$-direction and $y$-direction several times to avoid the rotor-stator rubbing. The practical feasibility of this approach is investigated by numerical simulations.

\section{Introduction}

Most machinery works with rotary elements or is powered by rotary machines such as electric motor and micromotor in microelectromechanical system. Forced vibrations in these systems are usually caused by inertia forces due to rotor mass imbalance and various transmission forces. If the vibration amplitude exceeds the gap between the rotor and the stator, the physical contact of the rotor with a stationary element will take place. The contact, commonly referred to as rubs, is a serious malfunction in rotating machinery that may lead to a machine catastrophic failure. Thus, it is not surprising that researchers, over the past decades, have put serious efforts towards understanding the phenomena and finding means of controlling such unwanted behaviors.

The literature on controlling the rotor-stator rub is vast. In some work about vibration control, the control forces are acted on the rotor using magnetic bearing, for example, [1$3]$. On the other hand, an active auxiliary bearing concept has been developed to actuate on the bearing housings and thereby indirectly on the rotor in opposition to magnetic bearings. Jiang et al. [4] developed a controller based on stability analysis of the synchronous annular rub to reduce the rub severity through the active auxiliary bearing. Chavez et al. [5] used sliding control and cross-coupled feedback in a rotor system to reduce the impact forces and to decrease the lateral and torsional vibrations. Ginzinger and Ulbrich [6] developed a two-phase control strategy to stabilize the rotor system in case of rub and effectively avoid backward whirling. References [7, 8] considered the concept of using an active auxiliary bearing operating with an active magnetic bearing system.

Despite a large number of theoretical and experimental investigations conducted so far, the phase characteristics of interacted rotor-stator system seem to be not sufficient. Usually the state variables are chosen as displacement and velocity; however, amplitude and phase are also key variables for describing the vibration. In our previous works $[9,10]$ proposed a way to reduce the vibration amplitude at resonance by modulating the phase relations between excitement and response. Moreover, the phase characteristic of a disk rubbing with a ring supported elastically was used to explain the mechanism of stiffness increase phenomenon [11]. In this work, based on the phase characteristic an impulsive control strategy is presented to eliminate the rub of the rotor-stator system. An impulsive control method, which is proved to be effective for smooth system, has been developed to stabilize the chaotic motions in a vibro-impact system [12].

This paper is organized as follows. The rotor-stator model is described in Section 2. The relation of the vibration energy 
and the phase difference between the vibration velocity and the excitement force is presented in Section 3. The phase characteristic of steady-state periodic solution is analyzed in Section 4. Based on the phase characteristic, the impulsive control strategy is obtained in Section 5. The practical feasibility of this approach is investigated by numerical simulations in Section 6.

\section{Mathematical Model}

The rotor-stator system is shown in Figure 1(a). A disk with mass $m$ is mounted at the midpoint of the weightless shaft with total stiffness $k_{r}$ and viscous $c$ and rotating at a constant speed of $\omega$. The mass center of the rotor is located at a distance $\tilde{e}$ from its geometrical center. The stator is considered to be rigid and modeled as the radial springs with stiffness $k_{s}$. The clearance between the rotor and the stator is denoted by $\gamma$. For directly expressing the phase difference between the excitement (the rotating motion) and the response (the whirling motion), a polar coordinate system is chosen, as shown in Figure 1(b). The center of stator is assumed as the origin of the coordinates. The current position of the rotor $O_{r}$ is given by the radius $\tilde{\rho}$ and the angle $\psi$. The phase angle of the imbalance force is $\varphi=\omega t . \widetilde{v}$ is the vibration velocity and $\beta$ represents the phase angle of the vibration velocity. When $\tilde{\rho} \geq \gamma$, the normal contact force and the friction force between the rotor and the stator are $F_{N}$ and $F_{T}=\mu F_{N}$, respectively, where $\mu$ is the friction coefficient.

The equations that govern the motion of the rotor-stator system in polar coordinate form can be formulated into nondimensional form as

$$
\begin{gathered}
\ddot{\rho}+\nu \dot{\rho}+\rho-\rho \dot{\psi}^{2}+\Theta f_{N}-e \omega^{2} \cos (\omega \tau-\psi)=0, \\
\rho \ddot{\psi}+2 \dot{\rho} \dot{\psi}+\nu \rho \dot{\psi}+\Theta \mu f_{N}-e \omega^{2} \sin (\omega \tau-\psi)=0, \\
f_{N}=k(\rho-1),
\end{gathered}
$$

where the dot denotes the derivatives with respect to nondimensional time $\tau$. We define $\Theta=1$ if $\rho \geq 1$ and $\Theta=0$ if $\rho<1$. The nondimensional parameters and variables are listed as follows:

$$
\begin{gathered}
\omega_{n}^{2}=\frac{k_{r}}{m}, \quad \tau=\omega_{n} t, \quad \rho=\frac{\tilde{\rho}}{\gamma}, \\
\nu=\frac{c}{2 \sqrt{k_{r} m}}, \quad e=\frac{\widetilde{e}}{\gamma}, \\
f_{N}=\frac{F_{N}}{k_{r} \gamma}, \quad k=\frac{k_{s}}{k_{r}}, \quad X=\frac{x}{\gamma}, \\
Y=\frac{y}{\gamma}, \quad v=\frac{\widetilde{v}}{\gamma} .
\end{gathered}
$$

\section{The Effect of the Excitement Phase on the Vibration Energy}

In this section, the relation of the vibration energy and the phase difference between the vibration velocity and the excitement force is shown.
To get analytical results, we introduce the following transformations:

$$
\frac{\rho \dot{\psi}}{\sqrt{\dot{\rho}^{2}+\rho^{2} \dot{\psi}^{2}}}=\sin \theta, \quad \frac{\dot{\rho}}{\sqrt{\dot{\rho}^{2}+\rho^{2} \dot{\psi}^{2}}}=\cos \theta,
$$

where $\theta$ is the angle between radial velocity and the vibration velocity $v$.

From the above transformations (3), we obtain

$$
\begin{aligned}
& \dot{X}=\dot{\rho} \cos \psi-\rho \dot{\psi} \sin \psi=\sqrt{\dot{\rho}^{2}+\rho^{2} \dot{\psi}^{2}} \cos (\psi+\theta), \\
& \dot{Y}=\dot{\rho} \sin \psi+\rho \dot{\psi} \cos \psi=\sqrt{\dot{\rho}^{2}+\rho^{2} \dot{\psi}^{2}} \sin (\psi+\theta),
\end{aligned}
$$

where $\psi+\theta=\beta$ is the phase angle of the vibration velocity.

When $\rho<1$, the vibration energy of the rotor is

$$
V=\frac{\dot{\rho}^{2}}{2}+\frac{\rho^{2} \dot{\psi}^{2}}{2}+\frac{\rho^{2}}{2}
$$

and its derivative with respect to $\tau$ is

$$
V^{\prime}=\dot{\rho} \ddot{\rho}+\rho \dot{\rho} \dot{\psi}^{2}+\rho^{2} \dot{\psi} \ddot{\psi}+\rho \dot{\rho} .
$$

Using (1), (6) is written as

$$
\begin{aligned}
V^{\prime}= & -\nu\left(\dot{\rho}^{2}+\rho^{2} \dot{\psi}^{2}\right)+\dot{\rho} e \omega^{2} \cos (\omega \tau-\psi) \\
& +\rho \dot{\psi} e \omega^{2} \sin (\omega \tau-\psi) .
\end{aligned}
$$

By virtue of (4), (7) is written as

$$
V^{\prime}=\sqrt{2 T}\left(e \omega^{2} \cos (\omega \tau-\beta)-\nu \sqrt{2 T}\right),
$$

where $T=\left(\dot{\rho}^{2}+\rho^{2} \dot{\psi}^{2}\right) / 2$ is the dimensionless kinetic energy and $\omega \tau-\beta=\phi$ is the phase difference between the vibration velocity and the excitement force. By (8), it can be concluded that the increase/decrease of vibration energy depends on the phase difference $\phi$ : the vibration energy can be increased only if $\cos (\omega \tau-\beta)>0$; namely, the phase difference satisfies $-\pi / 2<\phi<\pi / 2$ (or modulo $2 \pi$ ), otherwise the vibration energy will be decreased.

Vibration energy or amplitude depends on the difference between the vibration velocity and the excitement force. If the vibration velocity has an instantaneous change, the phase difference is changed accordingly, which makes the vibration energy increase or decrease and alters the motion of the rotor. This suggests the starting point for controlling the rotorstator rub by implementing impulse.

\section{The Phase Characteristic of Steady-State Periodic Solution}

For steady-state periodic solution, the characteristics of the phase difference between the excitement force and the response $\varphi-\psi$ are analyzed. It is noticed that the $\varphi-\psi$ represents $\varphi-\psi$ or $\bmod (\varphi-\psi, 2 \pi)$, which is in the scope $[0,2 \pi]$. 


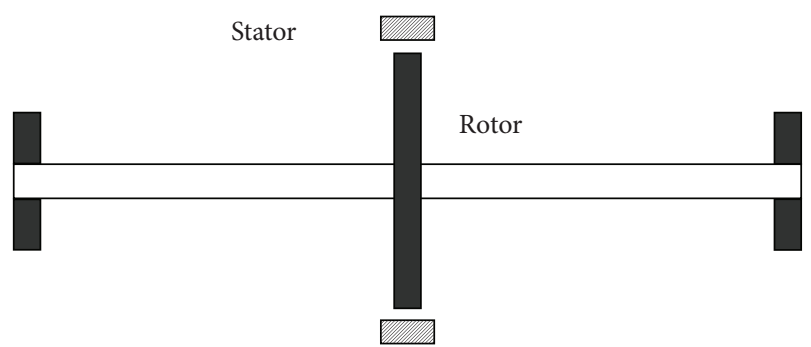

(a)

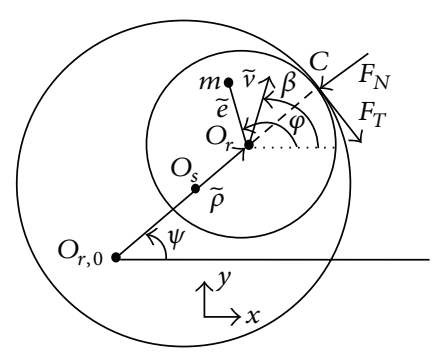

(b)

Figure 1: (a) The schematic plot of the rotor-to-stator system; (b) the section view on the plane of the rotor and the stator ring.

The rotor/stator system governed by (1) has a steady-state periodic solution that has constant amplitude and a frequency equal to the rotating speed of the rotor. The solution is corresponding to $\ddot{\rho}=\dot{\rho}=\ddot{\psi}=0$ and $\dot{\psi}=\dot{\varphi}=\omega$; substituting this specific solution into (1) yields

$$
\begin{gathered}
\rho-\rho \dot{\psi}^{2}+\Theta k(\rho-1)-e \omega^{2} \cos (\varphi-\psi)=0, \\
\nu \rho \dot{\psi}+\Theta \mu k(\rho-1)-e \omega^{2} \sin (\varphi-\psi)=0 .
\end{gathered}
$$

From (9), the radial amplitude $\rho$ and the phase difference $\varphi-\psi$ are obtained when $\Theta=0$ :

$$
\begin{gathered}
\left(\rho-\rho \omega^{2}\right)^{2}+(\nu \rho \omega)^{2}=\left(e \omega^{2}\right)^{2} \\
\tan (\varphi-\psi)=\frac{\nu \omega}{1-\omega^{2}} .
\end{gathered}
$$

When $\Theta=1$,

$$
\begin{aligned}
& \left(\rho-\rho \omega^{2}+k \rho-k\right)^{2}+(\nu \rho \omega+\mu k \rho-\mu k)^{2}=\left(e \omega^{2}\right)^{2}, \\
& e \omega^{2}\left[(\nu \omega+\mu k) \cos (\varphi-\psi)-\left(1+k-\omega^{2}\right) \sin (\varphi-\psi)\right] \\
& \quad=k\left(\mu-\mu \omega^{2}-\nu \omega\right) .
\end{aligned}
$$

The amplitude and the phase difference are expressed as a function of the rotor speed $\omega$ in (10) and (11). In (10), the nondimensional viscous $\nu$ is small; it is obvious that the phase difference $\varphi-\psi$ approaches to $\pi$ with the increase of the rotor speed $\omega$ when $\omega>1$. For synchronous full annular rub solution, the phase difference $\varphi-\psi$ is less than $\pi / 2$, which was explained in [11]. As an example, the phase difference and radial displacement depending on the rotor speed are numerically plotted according to (10) and (11) in Figures 2(a) and 2(b), respectively, for $\nu=0.1, \mu=0.06$, and $e=0.2, k=$ 8.

In Figure 2(a), the contact does not happen along the curve denoted by number 1 . The rotor first contacts the bearing at the rotor speed $\omega=0.925$ marked as number 2 where the curve has its first kink. It can be found that when the contact occurs, the phase approaches to $\omega=2.321$ marked by number 3 . At point 3 , an infinitesimal increase in rotor speed causes the phase difference jumping to point 5 , about $\pi$. Then the phase difference is kept along the curve marked by numbers 5-6. When the rotor speed is decreased, the phase difference follows the curve 6-5-4-2-1. A jump occurs at the point marked by number 4 , where the phase difference becomes less than $\pi / 2$ again. The corresponding radial amplitude diagrams are plotted in Figure 2(b); it can be seen that the amplitude jumps at the same speeds as the phase difference. Particularly, it is noticed that no-rub solution and rubbing solution coexist between the rotor speeds $\omega=1.103$ and $\omega=2.321$. The phase difference of the no-rub solution is about $\pi$, while the rubbing solution is less than $\pi / 2$, and the dashed curve represents unstable solution between the norub and rubbing solutions. $(\rho, \dot{\rho}, \psi, \dot{\psi})$ represents the position of rotor center; the phase difference $\varphi-\psi$ should be near $\pi / 2$ if $(\rho, \dot{\rho}, \psi, \dot{\psi})$ is in the attractive domain of the rubbing motion, while the phase difference $\varphi-\psi$ should be near $\pi$ if $(\rho, \dot{\rho}, \psi, \dot{\psi})$ is in the attractive domain of the no-rub motion.

\section{Impulsive Control Strategy}

Firstly, some basic knowledge about the impulsive control method is provided [12]. Suppose that the symbol $X_{i}(t)$ denotes the $i$ th state variable of a system. The technique of the method is that $X_{i}(t)$ performs changes in the form of instantaneous pulses, namely, in the form

$$
X_{i}^{*}(t)=(1-\lambda) X_{i}(t)
$$

where $\lambda$, which could be positive or negative, represents the strength of the pulse. In order to maintain the characteristic of the original system, the value of $|\lambda|$ cannot be too large. Therefore, the control is to make a tiny perturbation, with a proper value $\lambda$, to change the motions of the system.

According to the above section results, the increase/ decrease of vibration energy depends on the phase difference $\phi$. The vibration energy can be increased only if $\cos (\omega \tau-$ $\beta)>0$; namely, the phase difference satisfies $-\pi / 2<\phi<$ $\pi / 2$ (or modulo $2 \pi$ ). When the phase difference satisfies $\pi / 2<\phi<\pi$, the $\cos (\omega \tau-\beta)<0$; namely, the vibration energy will be decreased. Therefore, the control strategy in this paper is that the impulse is implemented in the radial and/or tangential direction occasionally to transit the phase difference $\phi$ towards $\pi / 2<\phi<\pi$ when the contact between the rotor and the stator occurs. The detail is given as follows. 


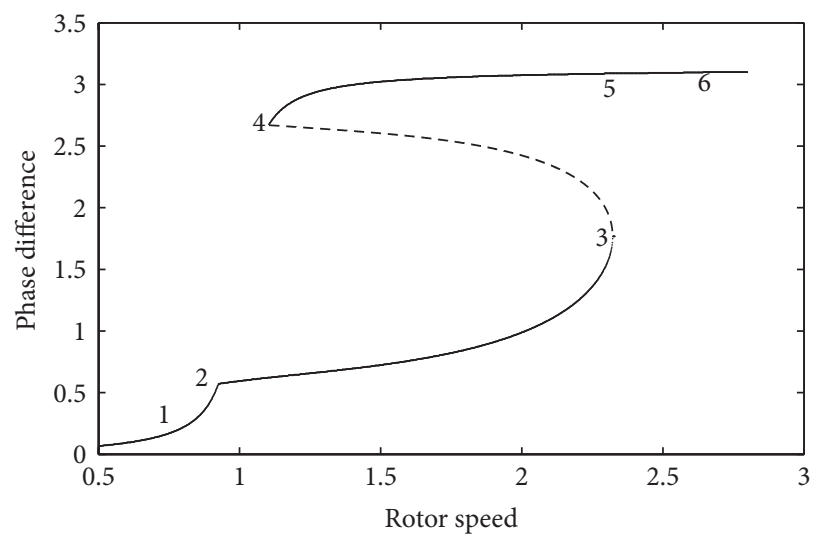

(a)

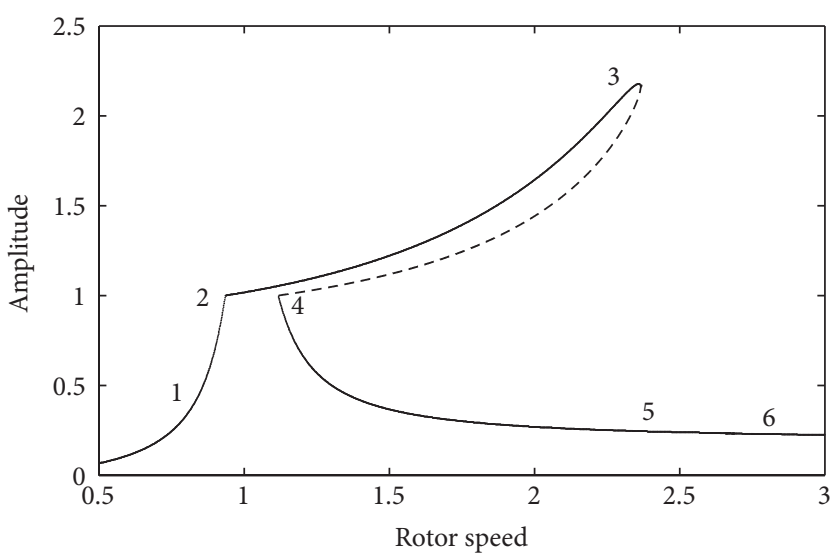

(b)

Figure 2: (a) Phase difference shift depending on rotor speed; (b) radial displacement amplitude depending on rotor speed.

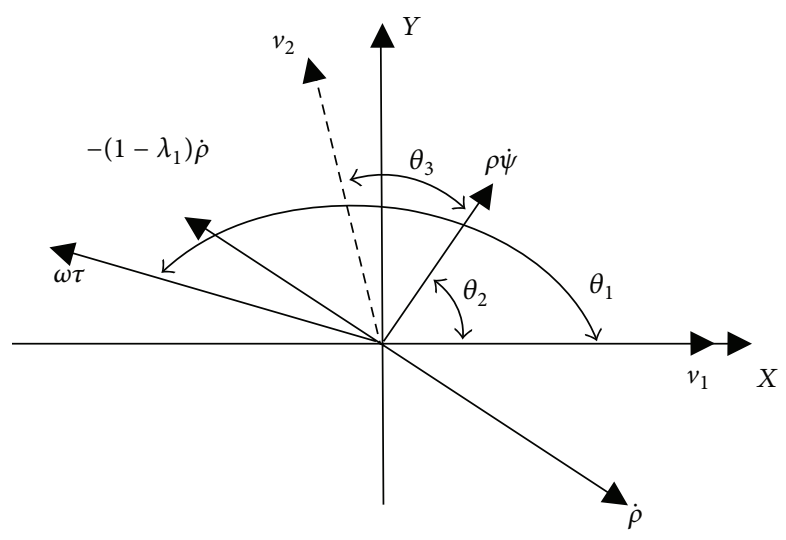

FIGURE 3: The schematic plot of the control strategy.

When $\rho<1$,

$$
\begin{gathered}
\ddot{\rho}+\nu \dot{\rho}+\rho-\rho \dot{\psi}^{2}=e \omega^{2} \cos (\omega \tau-\psi), \\
\rho \ddot{\psi}+2 \dot{\rho} \dot{\psi}+\nu \rho \dot{\psi}=e \omega^{2} \sin (\omega \tau-\psi) .
\end{gathered}
$$

When $\rho=1$,

$$
\begin{aligned}
\dot{\rho}^{*} & =-\left(1-\lambda_{1}\right) \dot{\rho}^{-}, \\
\rho \dot{\psi}^{*} & =-\left(1-\lambda_{2}\right) \rho \dot{\psi}^{-},
\end{aligned}
$$

where $\dot{\rho}^{-} \rho \dot{\psi}^{-} \dot{\rho}^{*} \rho \dot{\psi}^{*}$ represent the radial and tangential velocities, immediately before and after implementing impulse, respectively. When the contact between the rotor and the stator occurs, the proper parameters $\lambda_{1}$ and $\lambda_{2}$ are chosen to transit the phase difference $\phi$ towards $\pi / 2<\phi<\pi$.

When the contact between the rotor and the stator occurs, the phase difference $\phi$ locates in one of the four quadrants. For the different situation, we adopt different control strategy. For convenience, we introduce the following variables: $\theta_{1}$ is the phase difference $\phi$ before control; $\theta_{2}=\arctan (\rho / \rho \dot{\psi}) ; \theta_{3}=$ $\arctan \left(-\left(1-\lambda_{1}\right) \rho / \rho \dot{\psi}\right) ; \theta_{4}=\arctan \left(\left(1-\lambda_{1}\right) \rho /\left(1-\lambda_{2}\right) \rho \dot{\psi}\right)$.
When $-\pi / 2<\phi<0$, the impulse is implemented in radial direction, that is, $\dot{\rho}^{*}=-\left(1-\lambda_{1}\right) \dot{\rho}^{-}$, where $\lambda_{1}$ is chosen to ensure the following condition:

$$
\frac{\pi}{2}<\theta_{1}+\theta_{2}-\theta_{3}<\pi
$$

Then, the phase difference $\phi$ is transited into $[\pi / 2, \pi]$ after control.

When $0<\phi<\pi / 2$, the impulse is implemented in radial direction and tangential direction, that is, $\dot{\rho}^{*}=-\left(1-\lambda_{1}\right) \dot{\rho}^{-}$ and $\rho \dot{\psi}^{*}=-\left(1-\lambda_{2}\right) \rho \dot{\psi}^{-}$, where $\lambda_{1}$ and $\lambda_{2}$ are chosen to ensure the following condition:

$$
\frac{\pi}{2}<\theta_{2}+\pi-\theta_{4}-\theta_{1}<\pi
$$

Then, the phase difference $\phi$ is transited into $[\pi / 2, \pi]$ after control.

When $\pi / 2<\phi<\pi$, the impulse is implemented in radial direction, that is, $\dot{\rho}^{*}=-\left(1-\lambda_{1}\right) \dot{\rho}^{-}$, where $\lambda_{1}$ is chosen to ensure the following condition:

$$
\frac{\pi}{2}<\theta_{1}-\theta_{2}-\theta_{3}<\pi .
$$

Then, the phase difference $\phi$ is transited into $[\pi / 2, \pi]$ after control.

When $\pi<\phi<3 \pi / 2$, the impulse is implemented in radial direction, that is, $\dot{\rho}^{*}=-\left(1-\lambda_{1}\right) \dot{\rho}^{-}$, where $\lambda_{1}$ and $\lambda_{2}$ are chosen to ensure the following condition:

$$
\frac{\pi}{2}<\theta_{1}-\theta_{2}-\theta_{3}<\pi .
$$

Then, the phase difference $\phi$ is transited into $[\pi / 2, \pi]$ after control.

In order to understand the impulsive control strategy better, a diagram is given to illustrate one case of them. For the case of $\pi / 2<\phi<\pi$, the impulsive control strategy is shown as Figure 3 , where $v_{1}$ is the velocity of the rotor before impact and $v_{2}$ is the velocity of the rotor after impact.

In real operation, the impulse implemented in the radial and tangential directions is decompounded into horizontal and vertical directions, which make the method feasible. 


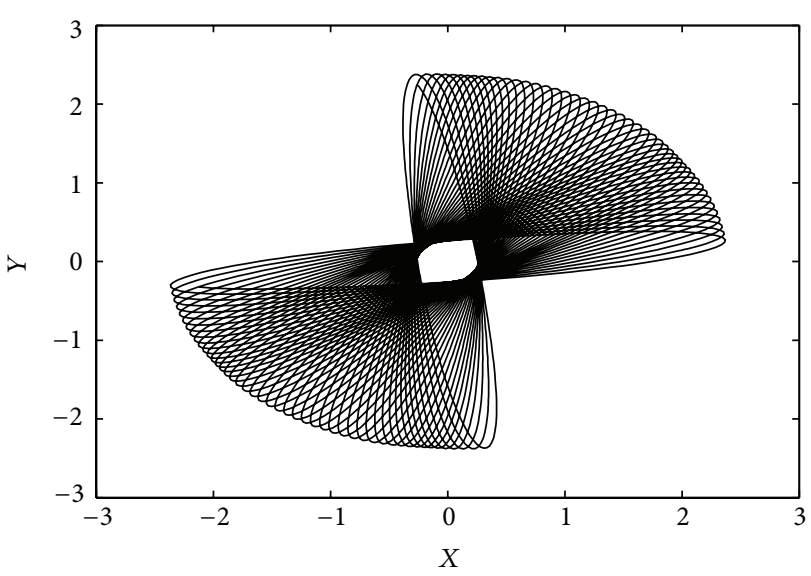

(a)

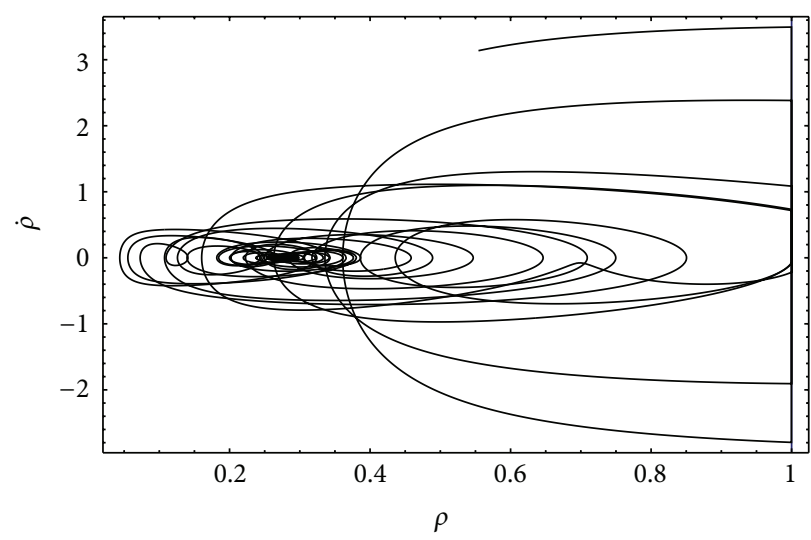

(c)

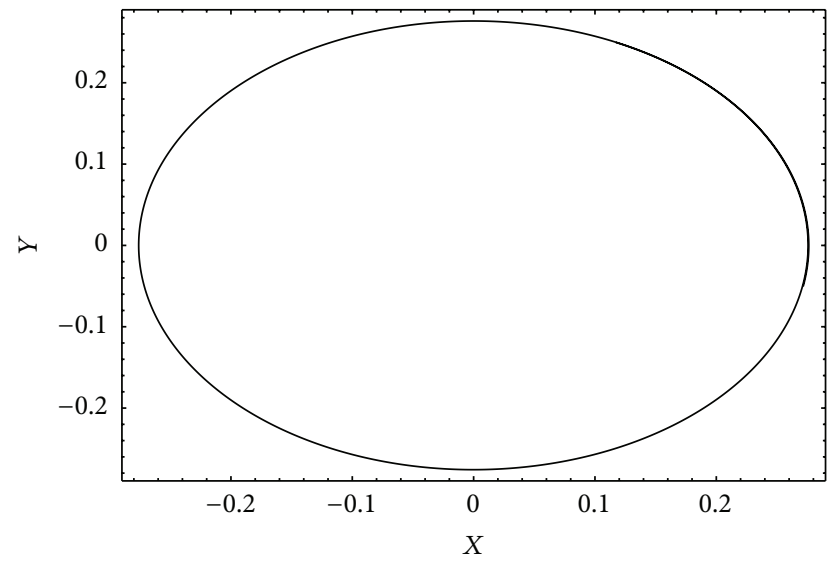

(b)

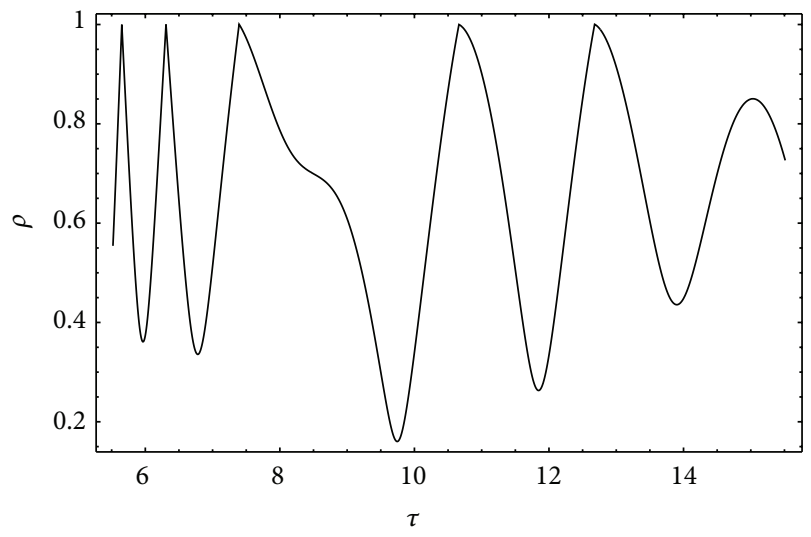

(d)

FIGURE 4: Control result at $\omega=1.9$. (a) The trajectory of rotor center before control; (b) the trajectory of rotor center after control; (c) the phase portrait of rotor center by impulsive control; (d) the time response of radial displacement by impulsive control.

\section{Numerical Simulations}

To investigate the practical feasibility of the above strategy, numerical simulation is carried out in this section.

The parameters of the rotor-stator system are chosen to be $\nu=0.1, \mu=0.06$, and $e=0.2$, and $k=8$.

For rotor speed $\omega=1.9$, the response of rotor is a partial rub with forward whirl as shown in Figure 4(a). According to the above discussion, for different $\phi$, the different impulsive control should be implemented. The detailed numerical simulations are given below:

$$
\begin{aligned}
& \text { If }-\pi / 2<\phi<0 \text {, choose } \lambda_{1}=0.2 ; \\
& \text { If } 0<\phi<\pi / 2 \text {, choose } \lambda_{1}=0.8, \lambda_{2}=0.2 ; \\
& \text { If } \pi / 2<\phi<\pi \text {, choose } \lambda_{1}=0.9 ; \\
& \text { If } \pi<\phi<3 \pi / 2 \text {, choose } \lambda_{1}=0.8
\end{aligned}
$$

Then, the partial rub with forward whirl is stabilized to no-rub motion as shown in Figure 4(b). The process of impulsive control is shown in Figures 4(c) and 4(d); when the contact between the rotor and the stator occurs, the impulse is implemented in the radial and/or tangential direction to transit the phase difference $\phi$ towards $\pi / 2<\phi<\pi$; after five collisions, the response of rotor is in no-rub motion.
For rotor speed $\omega=2.1$, the response of rotor is a partial rub with backward whirl as shown in Figure 5(a). The detailed numerical simulations are given below:

$$
\begin{aligned}
& \text { If }-\pi / 2<\phi<0 \text {, choose } \lambda_{1}=0.2 ; \\
& \text { If } 0<\phi<\pi / 2 \text {, choose } \lambda_{1}=0.8, \lambda_{2}=0.2 ; \\
& \text { If } \pi / 2<\phi<\pi \text {, choose } \lambda_{1}=0.9 ; \\
& \text { If } \pi<\phi<3 \pi / 2 \lambda_{1}=0.8 \text {. }
\end{aligned}
$$

Then, the partial rub with backward whirl is stabilized to no-rub motion as shown in Figure 5(b). The process of impulsive control is shown in Figures 5(c) and 5(d); after four collisions, the response of rotor is in no-rub motion.

\section{Conclusions}

In this paper, a novel concept, the impulsive control method based on the phase characteristic analysis, is proposed to overcome the problem of rotor-stator rub. Usually the state variables of rotor-stator system are chosen as displacement and velocity; however, amplitude and phase seem to be ignored although they are also key variables for describing the vibration. This paper chooses amplitude and phase as 


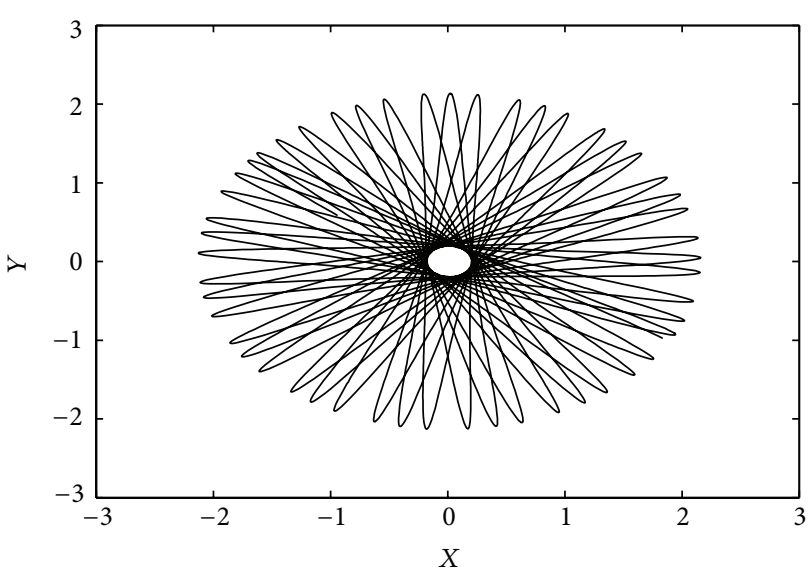

(a)

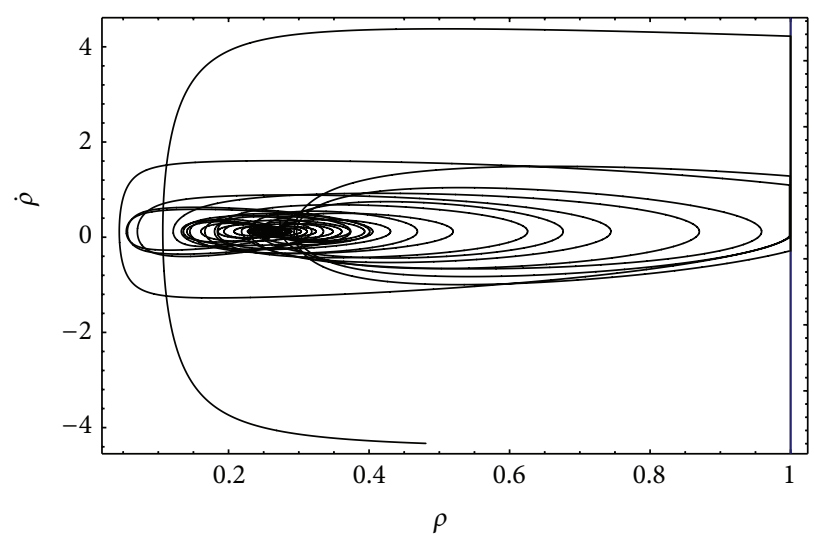

(c)

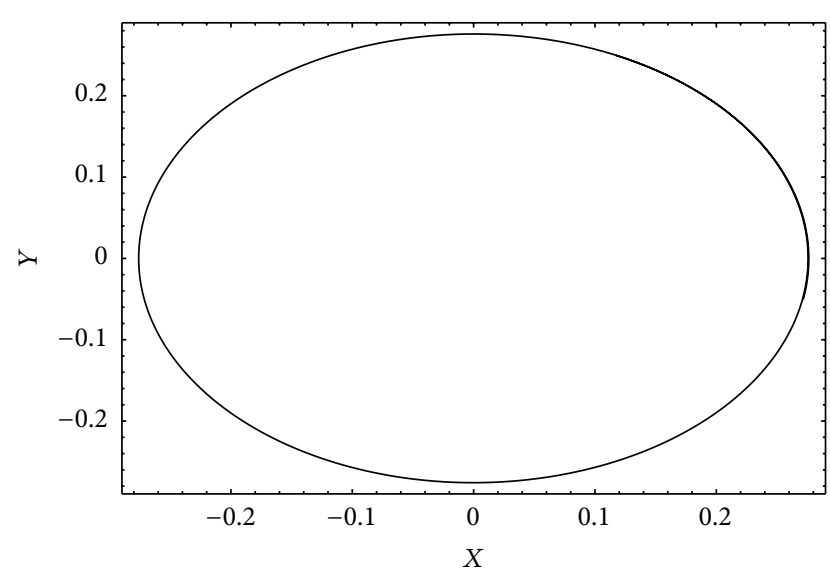

(b)

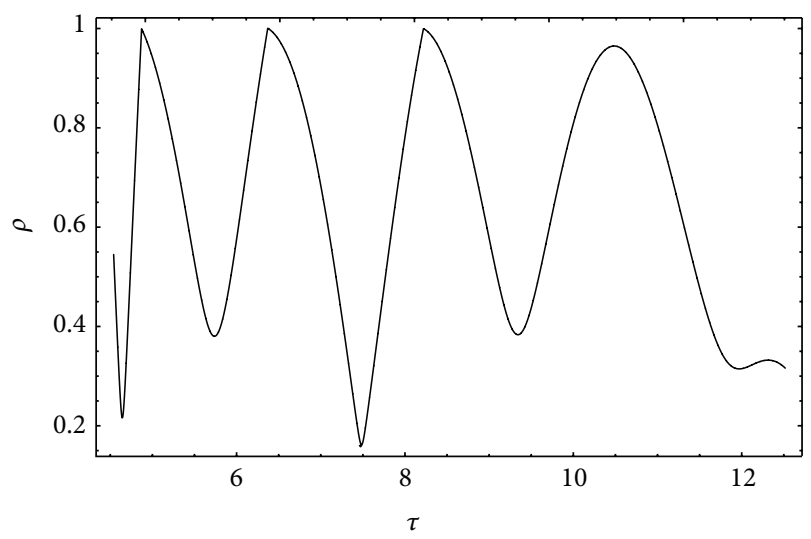

(d)

Figure 5: Control result at $\omega=2.1$. (a) The trajectory of rotor center before control; (b) the trajectory of rotor center after control; (c) the phase portrait of rotor center by impulsive control; (d) the time response of radial displacement by impulsive control.

the state variables and analyzes the phase characteristic of interacted rotor-stator system. Based on the obtained phase characteristic, an impulsive control strategy is presented to eliminate the rub of the rotor-stator system. The feasibility of this approach is investigated by numerical simulation. If this technique can be implemented in real machinery, it will be efficient since it only requires to know the phase difference of this system, and the control force can be determined definitely according to the phase difference. Future work will further explore the ideas for other systems and will also be concerned with the experimental validation of this control strategy.

\section{Conflict of Interests}

The authors declare that there is no conflict of interests regarding the publication of this paper.

\section{Acknowledgments}

This work is supported by the project sponsored by the National Natural Science Foundation of China (no. 11372077), the Guangxi University Foundation sponsored by the Comprehensive Strength Enhancement Project of Midwest Universities, and the Scientific Research Foundation of Guangxi University (no. XBZ120251).

\section{References}

[1] M. O. T. Cole and P. S. Keogh, "Asynchronous periodic contact modes for rotor vibration within an annular clearance," Proceedings of the Institution of Mechanical Engineers C: Journal of Mechanical Engineering Science, vol. 217, no. 10, pp. 1101-1115, 2003.

[2] M. O. T. Cole and P. S. Keogh, "Rotor vibration with auxiliary bearing contact in magnetic bearing systems-part 2: robust synchronous control for rotor position recovery," Proceedings of the Institution of Mechanical Engineers C: Journal of Mechanical Engineering Science, vol. 217, no. 4, pp. 393-409, 2003.

[3] M. Schlotter and P. S. Keogh, "Synchronous position recovery control for flexible rotors in contact with auxiliary bearings," Journal of Vibration and Acoustics, vol. 129, no. 5, pp. 550-558, 2007.

[4] J. Jiang, H. Ulbrich, and A. Chavez, "Improvement of rotor performance under rubbing conditions through active auxiliary 
bearings," International Journal of Non-Linear Mechanics, vol. 41, no. 8, pp. 49-957, 2006.

[5] A. Chavez, H. Ulbrich, and L. Ginzinger, "Reduction of contact forces in a rotor-stator-system in case of rubbing through active auxiliary bearing," Shock and Vibration, vol. 13, no. 4-5, pp. 505518, 2006.

[6] L. Ginzinger and H. Ulbrich, "Simulation-based controller design for an active auxiliary bearing," Journal of System Design and Dynamics, vol. 3, no. 4, pp. 607-616, 2009.

[7] I. S. Cade, M. N. Sahinkaya, C. R. Burrows, and P. S. Keogh, "An active auxiliary bearing control strategy to reduce the onset of asynchronous periodic contact modes in rotor/magnetic bearing systems," Journal of Engineering for Gas Turbines and Power, vol. 132, no. 5, Article ID 052502, 9 pages, 2010.

[8] I. S. Cade, M. N. Sahinkaya, C. R. Burrows, and P. S. Keogh, "On the use of actively controlled auxiliary bearings in magnetic bearing systems," Journal of Engineering for Gas Turbines and Power, vol. 131, no. 2, Article ID 022507, 10 pages, 2009.

[9] S. M. Wang, Q. S. Lu, and Q. Wang, "Reducing the amplitude of vibration at resonances by phase modulation," Journal of Sound and Vibration, vol. 290, no. 1-2, pp. 410-424, 2006.

[10] S. M. Wang, Q. S. Lu, and E. H. Twizell, "Reducing lateral vibration of a rotor passing through critical speeds by phase modulating," Journal of Engineering for Gas Turbines and Power, vol. 125, no. 3, pp. 766-771, 2003.

[11] S. M. Wang, J. Q. Xu, and Q. Wang, "The mechanism of stiffness increase phenomenon of a rubbing disk," Acta Mechanica Sinica, vol. 26, no. 3, pp. 441-448, 2010.

[12] L. Wang, W. Xu, and Y. Li, "Impulsive control of a class of vibroimpact systems," Physics Letters A, vol. 372, no. 32, pp. 53095313, 2008. 


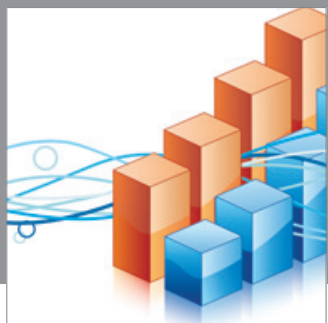

Advances in

Operations Research

mansans

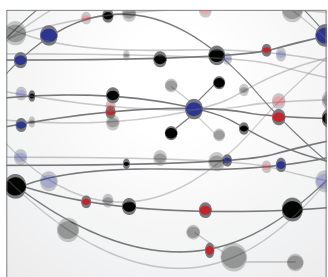

The Scientific World Journal
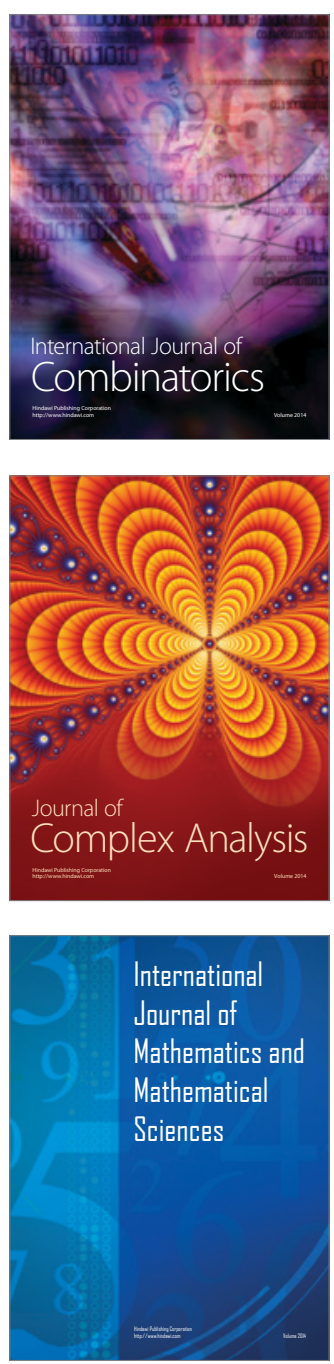
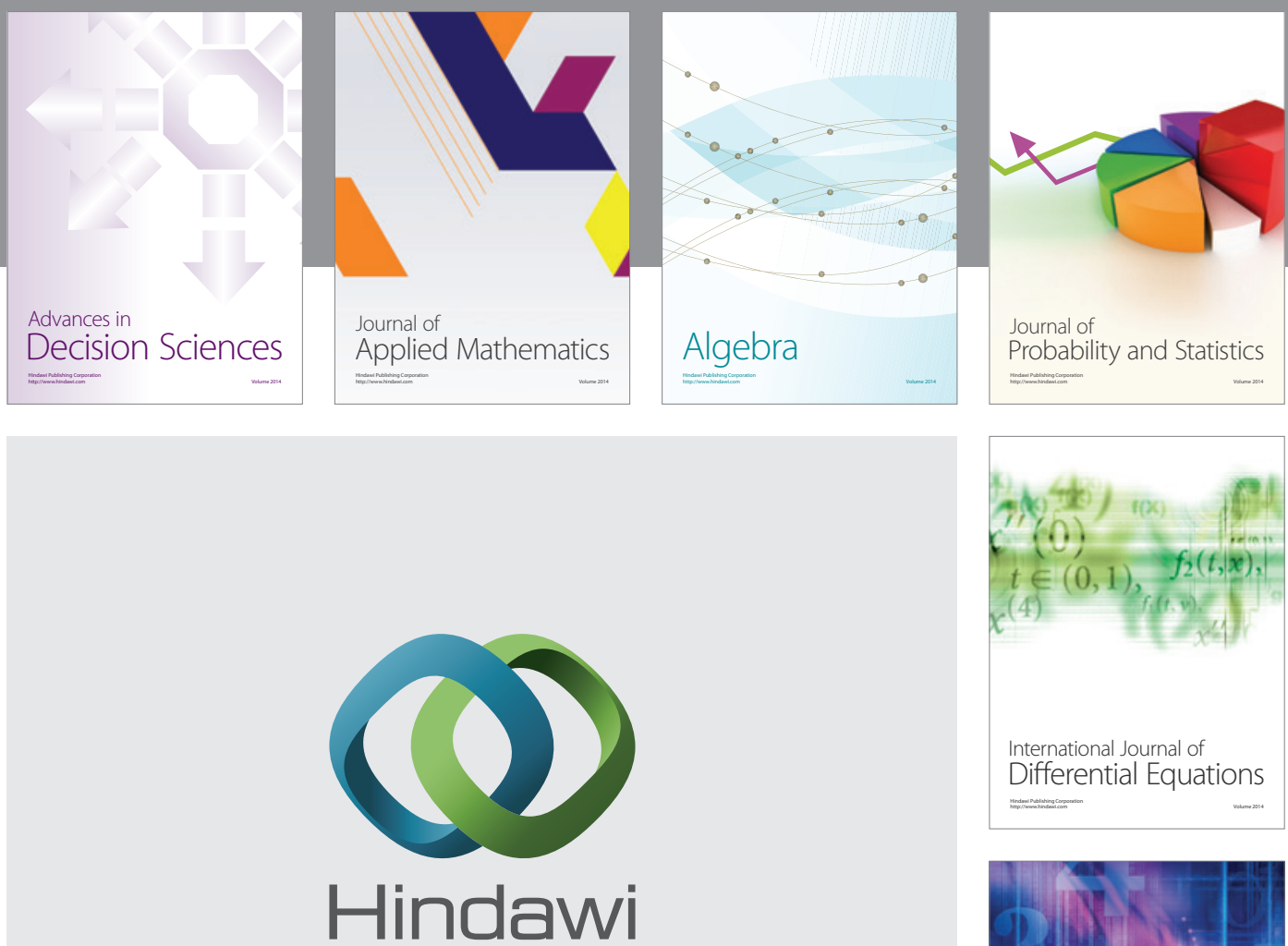

Submit your manuscripts at http://www.hindawi.com
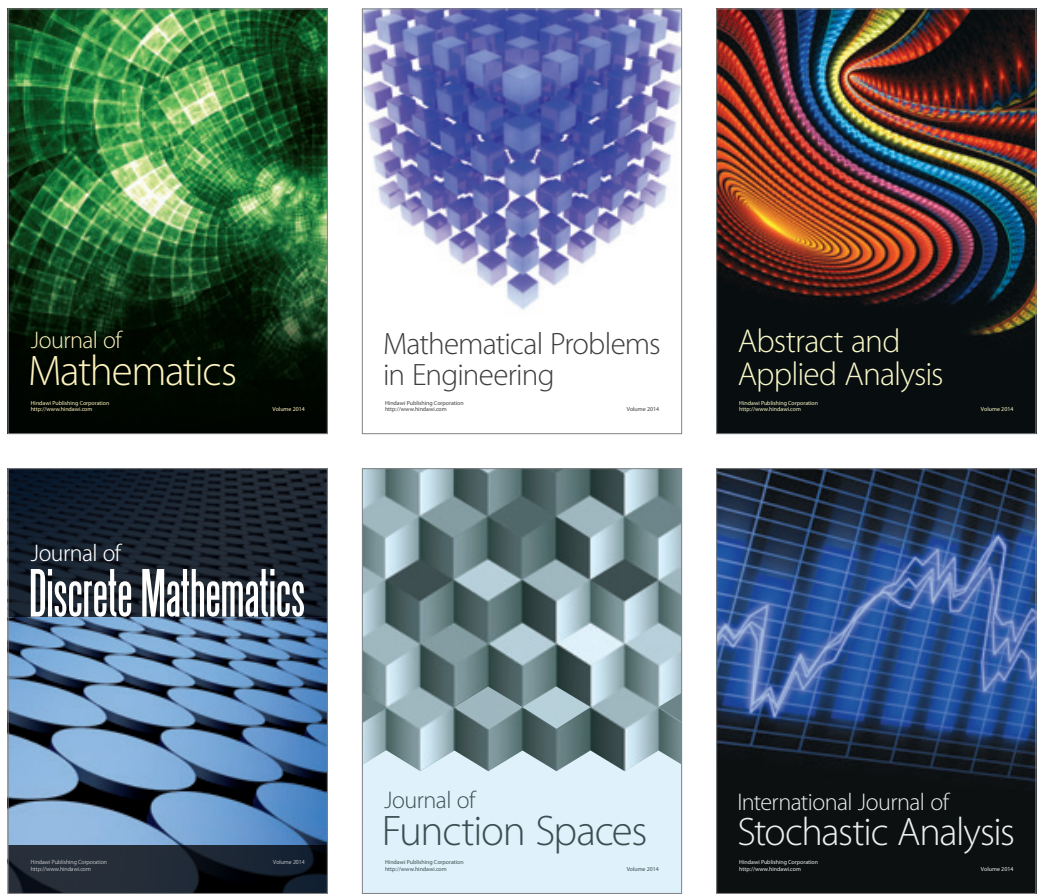

Journal of

Function Spaces

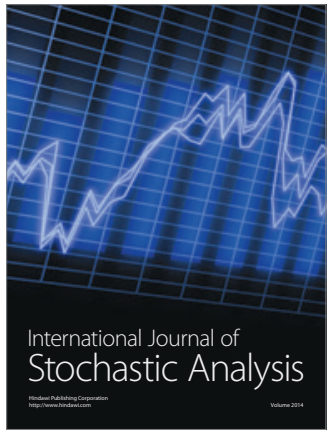

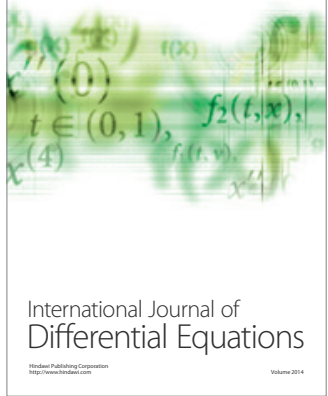
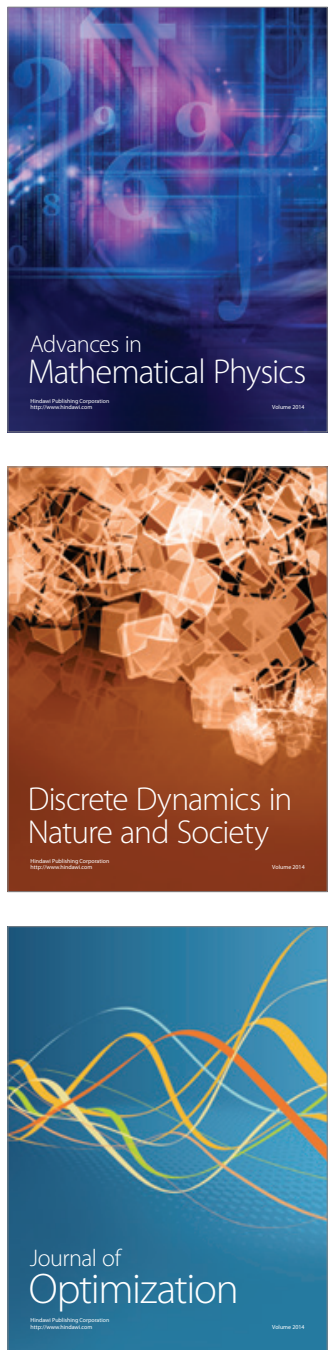